\title{
Submicron YBaCuO biepitaxial Josephson junctions: $d$-wave effects and phase dynamics
}

\author{
D. Stornaiuolo, ${ }^{1,2, a)}$ G. Rotoli, ${ }^{2}$ K. Cedergren, ${ }^{3}$ D. Born, ${ }^{4}$ T. Bauch, ${ }^{3}$ F. Lombardi, ${ }^{3}$ and \\ F. Tafuri ${ }^{1,2}$ \\ ${ }^{1}$ CNR-SPIN, 80126 Napoli, Italy \\ ${ }^{2}$ Dipartimento di Ingegneria dell'Informazione, Seconda Università di Napoli, 81031 Aversa (CE), Italy \\ ${ }^{3}$ Department of Microtechnology and Nanoscience, Quantum Device Physics Laboratory, Chalmers \\ University of Technology, SE-412 96 Goteborg, Sweden \\ ${ }^{4}$ NEST and Scuola Normale Superiore, 56126 Pisa, Italy and Dipartimento di Ingegneria dell'Informazione, \\ Seconda Università di Napoli, 81031 Aversa (CE), Italy
}

(Received 24 February 2010; accepted 14 March 2010; published online 1 June 2010)

\begin{abstract}
We report a systematic study of the transport properties of high critical temperature superconductor (HTS) biepitaxial Josephson junctions in the submicron range. Junction performances point to more uniform and reproducible devices and to better control of $d$-wave intrinsic properties. Outcomes promote novel insights into the transport mechanisms across grain boundaries and encourage further developments in the control of dissipation in HTS devices. The application of nanotechnology to HTS could be an additional tool to properly engineer the junction properties to match specific circuit design also in view of the integration into hybrid quantum circuits. (c) 2010 American Institute of Physics. [doi:10.1063/1.3388035]
\end{abstract}

\section{INTRODUCTION}

Grain boundary (GB) junctions have played a crucial role in investigating the physics of high critical temperature superconductors (HTS). ${ }^{1-3}$ The study of the $d$-wave order parameter (OP) symmetry is probably one of the most remarkable example ${ }^{1}$ and its impact on the analysis of unconventional aspects of the Josephson effect is impressive.

More recently, YBaCuO GB biepitaxial (BP) Josephson junctions (JJs) have been used to prove for the first time macroscopic quantum effects in an HTS device. ${ }^{4,5}$

The existence of macroscopic quantum tunnel and energy level quantization effects in the proper HTS junction configuration argue against the common belief that the high level of dissipation induced by low energy quasiparticles in correspondence of the nodes of HTS OP would spoil the macroscopic coherence. It also brings to reconsider the intrinsic dissipation in these devices and their limits for the application to quantum circuitry. ${ }^{5,6}$ In the future, experiments aimed at investigating coherence times through time resolved measurements ${ }^{7}$ will provide an estimation of the ultimate limit for HTS JJs quantum performances. Despite the standards of low critical temperature superconductor (LTS) JJs still appear too challenging for HTS, various experiments, including MQT, have given indications that the gap in the performances between LTS and HTS may narrow in the future. In the long run novel strategies might allow to realize hybrid devices combining the different functionality of LTS and HTS. These targets can be achieved only through suitable junctions configurations, and $\mathrm{BP}$ junctions might play a relevant role thanks to their low transmission barriers and the possibility to exploit atomically flat interfaces. ${ }^{8}$ In these devices, high selectivity along the transport direction has also

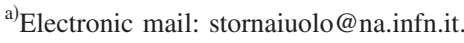

been demonstrated, ${ }^{9}$ which is one of the main ingredients to preserve intrinsic $d$-wave effects and coherence by imposing only few selective active channels. Moreover, BP junctions have the flexibility required for the integration in realistic hybrid circuits, as opposed to intrinsic junctions, where MQ effects have also been observed. ${ }^{10}$ A necessary step is now the fabrication of highly uniform and reproducible devices.

Many studies have shown that the disordered barrier microstructure is among the main causes responsible for HTS junctions low reproducibility and properties unpredictability. ${ }^{2,3}$ A random distribution of facets along the GB interface is for instance a well-known source of disorder. While some properties would be robust with respect to faceting and the presence of impurities along the barriers and in the electrodes, coherent behaviors of single transport channels would be averaged out by a relatively wide distribution of transport modes.

A possible strategy toward more uniform and reproducible junctions passes through the realization of submicronand nano-structures where only a controlled limited number of facets find place. The ultimate goal would be a junction composed of only one facet where extrinsic sources of noise would be dramatically reduced. This state-of-the-art HTS nanodevice will represent a compromise between the highest potentials of nanotechnology and the limits imposed by the vulnerability of HTS, yet it will benefit from the advantages of HTS and of their unconventional superconductivity. ${ }^{1}$

The present work is a first systematic study on submicron BP Josephson devices. We demonstrate that significant improvements in terms of properties reproducibility can be obtained scaling down the junctions dimensions to the submicron range. The current versus voltage $(I-V)$ characteristics studied in this work show features (such as a reproducible hysteretic behavior and step structures at finite voltages) which point to a low dissipative nature of the junction and to 


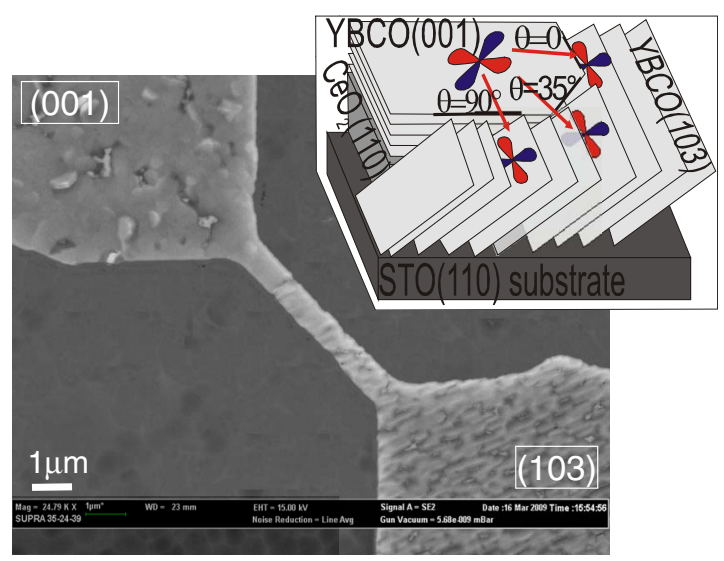

FIG. 1. (Color online) SEM image of a $0.8 \mu \mathrm{m}$ wide junction. In the inset a sketch of the junctions' structure is shown.

more controlled transport processes. The analysis of the switching process from the superconducting branch to the normal state at voltage value $\Delta_{s w}$ has been also carried out, suggesting a correlation of $\Delta_{s w}$ with the interface orientation and the $d$-wave OP profile. Neat fingerprints of the resistively and capacitively shunted junction (RCSJ) model are found. The results add novel information to the few studies realized on submicron HTS junctions, mostly bicrystal, available in literature, ${ }^{11-14}$ and give promise of a new generation of competitive deep-submicron GB HTS JJs.

\section{EXPERIMENTAL DETAILS}

In the off-axis BP devices described in this work, the junction barriers were created taking advantage of the epitaxial relations among $\mathrm{YBa}_{2} \mathrm{Cu}_{3} \mathrm{O}_{7-x}$ (YBCO), a $\mathrm{SrTiO}_{3}$ (STO) substrate and a $\mathrm{CeO}_{2}$ seed layer, ${ }^{9,15}$ as sketched in the inset of Fig. 1. The fabrication process is described in detail elsewhere, ${ }^{16}$ here we will only outline the main concepts. First, a thin $\mathrm{CeO}_{2}$ film was deposited using pulsed laser deposition (PLD) on the STO (110) oriented substrate, adjusting the deposition conditions in order to have a fully oriented (110) film, and then patterned using electron beam lithography and Ar ion milling. A YBCO film was subsequently deposited on the structured substrate using again PLD. The YBCO grows following the (103) orientation on the substrate and the (001) one on the seed layer area, thus generating a GB where the two orientations meet. The submicron bridges were defined using again electron beam lithography based on a multilayer carbon/PMMA (polymethyl methacrylate) mask. ${ }^{11,14,16,17}$ After exposing and developing the PMMA, the pattern was transferred first to an evaporated chromium film using lift off, then to the carbon layer using reactive ion etching. The patterned carbon layer will then act as a mask for the shaping of the YBCO film by Ar ion beam etching. In Fig. 1 we show a scanning electron microscope (SEM) image of a $0.8 \mu \mathrm{m}$ device obtained using this process. Thanks to the presence of a seed layer, various interface configurations can be realized on the same chip (three of them are indicated with different values of the misalignment angle $\theta$ in the inset of Fig. 1). The transport properties of the junctions were investigated down to $0.3 \mathrm{~K}$ using a shielded Heliox $3 \mathrm{He}$ refrigerator. A few measurements were taken also in a dilution refrigerator down to $30 \mathrm{mK}$. Both cryogenic inserts have been equipped with Resistor-Capacitor (RC) filters with a cut-off frequency of $1.6 \mathrm{MHz}$ and lowpass copper-powder filters with a cut-off frequency of 10 $\mathrm{GHz}$ for the filtering of electrical noise. The $I-V$ characteristics of the junctions were measured by using a four terminal configuration.

\section{RESULTS AND DISCUSSION}

This work was inspired by the experiment we have performed in the past on junctions a few micrometers wide (above $4 \mu \mathrm{m}){ }^{9}$ which clearly sets as a reference test. The comparison between the two experiments suggests relevant advances in the performances of the new submicron junctions and novel insights on the study of transport processes in HTS JJs. We have recently shown, in a work focused on the fabrication process, ${ }^{16}$ how submicron junctions properties, once the e-beam and ion milling procedures parameters have been fixed, critically depends on the width of the junction and the thickness of the YBCO film. Here we focus on $0.8 \mu \mathrm{m}$ wide junctions realized with $200 \mathrm{~nm}$ thick YBCO. These parameters represent our current limit for a top-down approach for high quality reproducible devices with a yield above $85 \%$. They also signal a passage to a regime where properties are not mediated over a large number of facets macroscopically different from each other, as occurring in junctions a few microns wide, but rather result from an average on two-three facets, with a more intrinsic behavior as an obvious consequence. This result is fully consistent with experiments on bicrystal junctions (with misorientation angles of $0 / 32^{\circ}, 0 / 40^{\circ}$, and $\left.0 / 45^{\circ}\right),{ }^{11}$ which have given indications of a crossover between two distinct transport regimes occurring at about one micron. The submicron regime has been interpreted by Tzalenchuck et al. ${ }^{11}$ as representative of the intrinsic nature of the barrier. An exact determination of the threshold width to observe intrinsic behavior is something that goes beyond the aims of this work. However we feel that a definitive word on this size threshold and on the meaning of what can be really considered intrinsic for an HTS junction will be probably given only the day we have reproducible and scalable devices down to the characteristic lengths of HTS systems.

\section{A. I-V characteristics}

All the submicron junctions investigated show a large hysteresis, see for example the $I-V$ characteristics showed in Fig. 2. The amplitude of the hysteresis is typically $50 \%$ of the total critical current $\left(I_{C}\right)$ at $\mathrm{T}=300 \mathrm{mK}$, with maximum values up to $75 \%$. Hysteresis in $I-V$ curves can be a measure of the incidence of capacitive effects in the junction, as described in the framework of the RCSJ model. ${ }^{18}$

The presence of well developed hysteresis allows for an unambiguous identification of the voltage value $\Delta_{s w}$ at which the current switches from the superconducting branch to the normal state. In LTS tunnel junctions $\Delta_{s w}$ and the $I_{C} R_{N}$ values and are neatly connected with the gap value $\Delta$, consistently with theory. ${ }^{18}$ Hysteretic $I-V$ curves in HTS junctions, on the contrary, are rare in literature and the switching volt- 

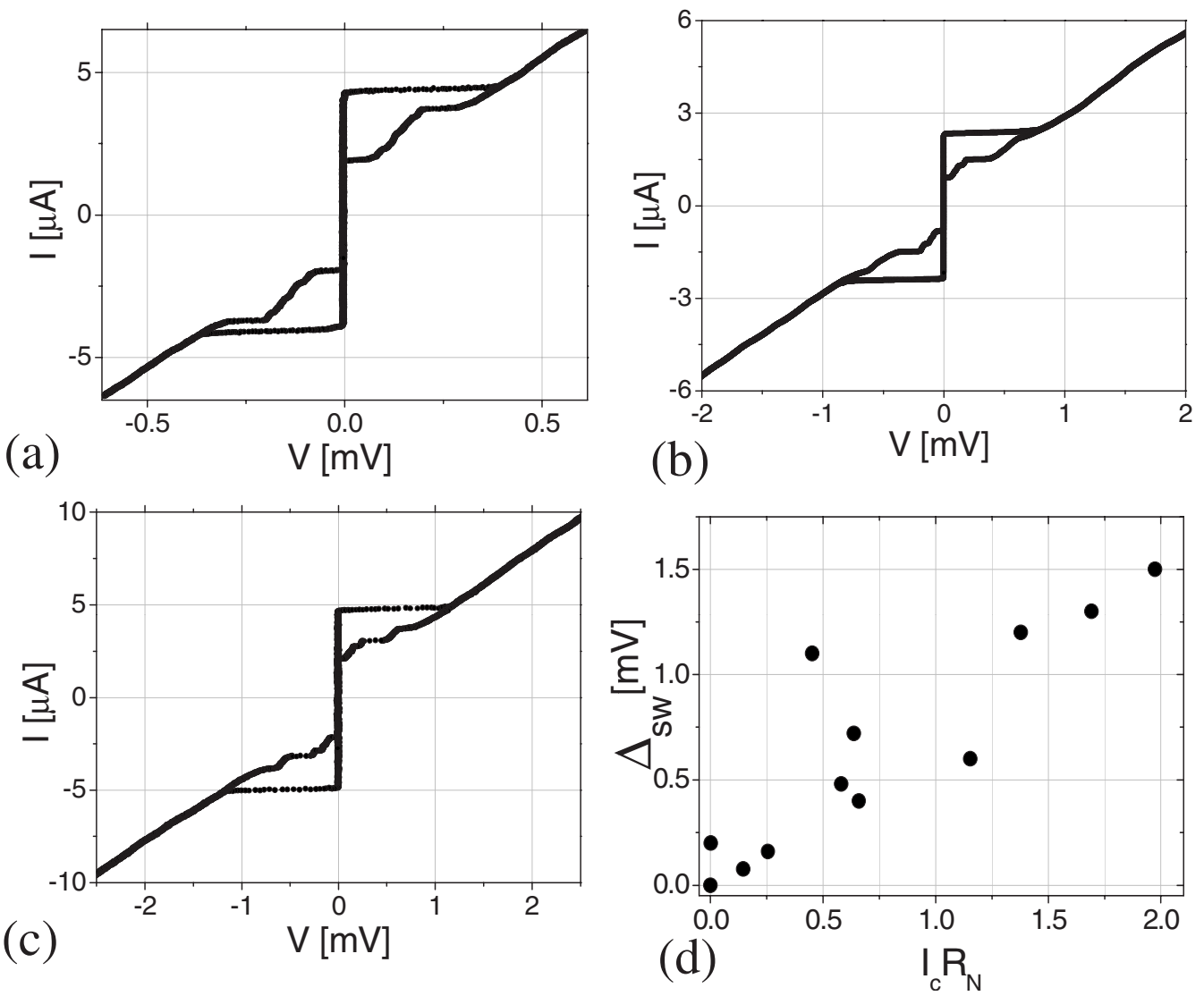

FIG. 2. $I-V$ characteristic of $0.8 \mu \mathrm{m}$ wide junctions with various misorientation angles: (a) $\theta=15^{\circ}$, (b) $\theta=40^{\circ}$, and (c) $\theta=50^{\circ}$. All the $I-V$ 's were measured with no applied magnetic field and at $\mathrm{T}=300 \mathrm{mK}$, except (b), which was measured at $\mathrm{T}=30 \mathrm{mK}$. Panel (d) shows the linear relation between the switching voltage $\Delta_{s w}$ and the $I_{C} R_{N}$ product of representative junctions.

age $\Delta_{s w}$ up to now has not been correlated with any specific scale energy. In our measurements $\Delta_{s w}$ scales linearly with the $I_{C} R_{N}$ value of the junctions up to $2 \mathrm{mV}$ [Fig. 2(d)], and a qualitative correlation between $I_{C} R_{N}$ and $\Delta_{s w}$ emerges for the first time also for HTS JJs, i.e., $I_{C} R_{N} \approx \Delta_{s w}$. This is fully consistent with the $I_{C} R_{N}$ values expected from the RCSJ model, which are typically one order of magnitude lower than the nominal gap values in the antinodal direction, and indicates that the switching processes are related to capacitive effects. We emphasize also that in LTS S-N-S junctions the appearance of hysteresis has been explained, within the RCSJ model, through an effective capacitance proportional to a diffusion time, which in turn is inversely proportional to the Thouless energy. ${ }^{19,20}$ This has remarkable similarities with the conclusions of one our previous works. ${ }^{21}$

\section{B. $I_{C} R_{N}$ versus junction angle dependence}

In the inset of Fig. 3 the modulation of the critical current with the interface orientation $\theta$ is reported. This modulation is a direct consequence of the $d$-wave symmetry of the OP. When compared with similar measurements on the same type of BP junctions on the scale of several microns ${ }^{9}$ (dashed line in the inset of Fig. 3), we notice for the $I_{C}$ a closer resemblance to the standard Sigrist-Rice formula. Moreover, a substantially angle independent $R_{N}$, which provides specific resistance values $R_{N} A$ of the order of $5 \times 10^{-7} \Omega \mathrm{cm}^{2}$, favors a remarkably agreement with the $d$-wave expectations also for the $I_{C} R_{N}$ versus $\theta$ dependence, which has never been observed up to now. ${ }^{9,22}$ We believe that $R_{N}$ is made homogeneous in the narrower barrier composed of only few facets, where the scattering is more uniform as well as the unavoidable damages caused during the fabrication process.

The $d$-wave profiles of $I_{C} R_{N}$ and $I_{C}$ are additional evidence that submicron scaling gives access to a more "intrinsic" nature of GB junctions. Since $I_{C} R_{N} \approx \Delta_{s w}, \Delta_{s w}$ is also modulated by $\theta$ according to the $d$-wave OP symmetry. This suggests the existence of a characteristic energy scale $E_{s c}$ $\approx I_{C} R_{N} \approx \Delta_{s w}$ for each interface configuration, determined by

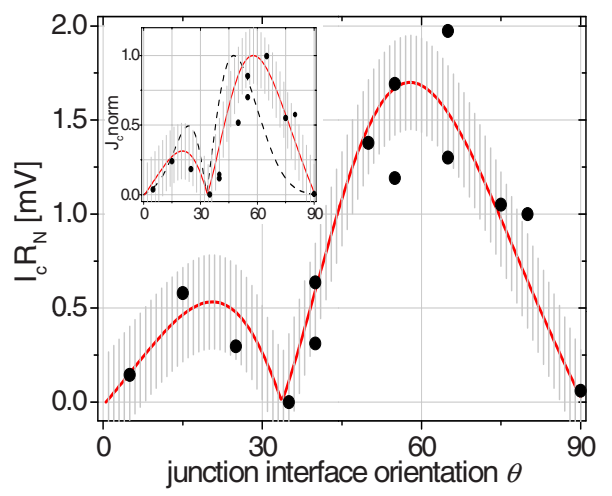

FIG. 3. (Color online) $I_{C} R_{N}$ product and $J_{C}$ values normalized to the maximum value (inset) of submicron junctions as a function of the misorientation angles. The comparison with results on junctions a few microns wide (dashed line in the inset) (Ref. 9) gives a quantitative information on a closer correspondence with the ideal Sigrist-Rice formula for off-axis biepitaxial junctions (solid line). 


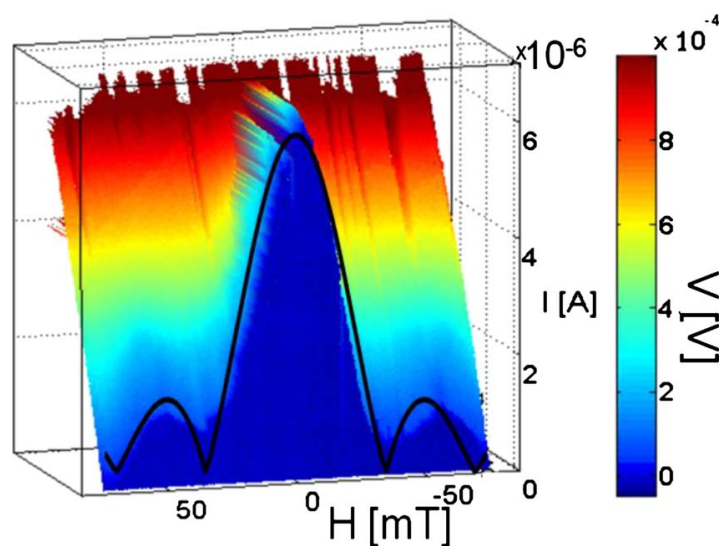

FIG. 4. (Color online) Three-dimensional plot of an experimental $I_{c}(H)$ pattern of a $0.8 \mu \mathrm{m}$ junction $\left(\theta=50^{\circ}\right)$ measured at $\mathrm{T}=0.3 \mathrm{~K}$. The superimposed black line is the Fraunhofer pattern.

the barrier orientation with respect to the $d$-wave profiles of the two electrodes. Further studies are required to establish how far this energy can be also related to characteristic mesoscopic energies. ${ }^{21}$

\section{Magnetic patterns}

The barrier uniformity is further testified by the regularity of most of the magnetic patterns of the submicron junctions, an example of which is shown in Fig. 4. The presence of one main central lobe in the $I_{C}(H)$ pattern, the ratio between the height of the central and the lateral lobes recall the Fraunhofer shape ${ }^{18}$ (thick black line in Fig. 4) which is theoretically expected for a perfect, defect-free and uniform barrier. This is another strong indication that the transport takes place in only few dominant facets, as opposed to several micron-wide GB junctions where the many facets, combined with the $d$-wave symmetry of the OP, lead to spontaneous fields along the GB and to anomalous $I_{C}(H)$ dependence. ${ }^{23}$

In planar thin film GB JJs, the effective area scales as the square of the width $\mathrm{L}$ rather than the usual $\mathrm{L}\left(\lambda_{1}+\lambda_{2}+\mathrm{t}\right)$ dependence of sandwich-type JJs (Ref. 24) (where $\lambda_{1}$ and $\lambda_{2}$ are the London penetration depth of the two electrodes and $t$ is the barrier thickness). Therefore, from the magnetic period, taking into account a London penetration depth of a few microns, ${ }^{4}$ we can infer an effective width very close to the nominal one of about $800 \mathrm{~nm}$, which leads to a critical current density of about $1 \times 10^{4} \mathrm{~A} / \mathrm{cm}^{2}$. These values are on average higher than those measured in micron-sized junctions and well match with a configuration characterized by reduced faceting.

\section{Resonant structures in the $I-V$ characteristics}

The presence of resonance steps at finite voltages in the $I-V$ characteristics, as it can be seen in panels of Fig. 2(a)-2(c) is another common feature of the BP submicron junctions, independently of the interface orientation. These steps in the $I-V$ curves signal fluxon dynamics of extended vortices in confined geometries. Step features are commonly used as fingerprints of subtle processes in LTS JJs. ${ }^{18,25-28}$ In HTS JJs resonances have been observed since the beginning and related to Fiske and Eck modes both in bicrystal and BP

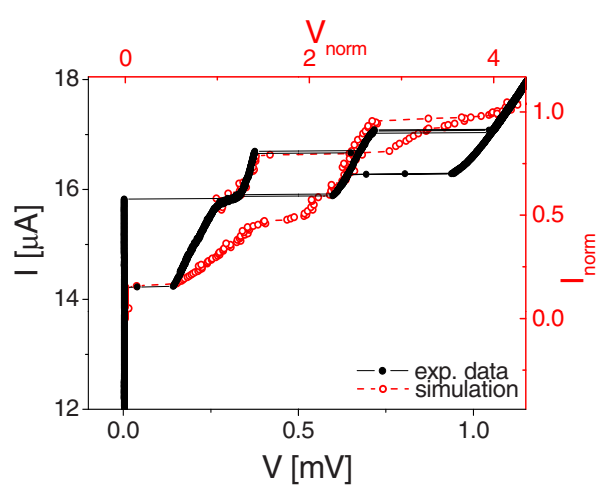

FIG. 5. (Color online) $I-V$ characteristic of a $0.8 \mu \mathrm{m}$ wide junction with a misalignment angle $\theta=65^{\circ}$, measured at $\mathrm{T}=0.3 \mathrm{~K}$ and $H=0$ (black dots). This $I-V$ was recorded with various amplitudes of the current sweep to visualize the intermediate states between 0 and $1 \mathrm{mV}$ and obtain a better resolution of the steps. A simulation made using a two facet configuration, $l=1.5$ and $\alpha=0.28$ is also shown (hollow grey dots, red online). For this simulation, the current is normalized to the critical current $I_{0}$ and the voltage to $\omega_{J} \Phi_{0} / 2 \pi$

junctions. They have played a relevant role in associating a dielectric behavior to the GB region. ${ }^{2,3}$ Along the GB line nonlinear waves would localize, and their standing or propagating nature would have been defined by boundary conditions, by the length of the actual GB interface and by the presence of faceting. Later, with a neater identification of induced $d$-wave effects, zero field steps (ZFS) in HTS have also been introduced. ${ }^{29}$ These appear in the $I-V$ characteristic similar to classical integer ZFS. ${ }^{18,30}$ The steps observed in the $I-V$ characteristics presented here prove that there is no intrinsic motivation to prevent the observation of very sharp resonances in HTS structures. The reason for the high dissipation commonly reported in these devices might be searched in extrinsic factors, such as impurities and lack of uniformity along the GB, rather than in the GB itself. In our submicron junctions low order resonances usually appear in the range of a few hundreds of microvolts, both in zero magnetic field and in presence of magnetic field. In order to obtain a better resolution of the steps intermediate states, we performed measurements with various amplitudes of the current sweep. One example is shown in Fig. 5 (black dots). This $I-V$ plot refers to a junction with a misalignment angle $\theta=65^{\circ}$ and $I_{C} R_{N}=1.3 \mathrm{mV}$, measured in absence of externally applied magnetic field. Two steps appear at 360 and $700 \mu \mathrm{V}$. These resonances are exceptionally clean for a HTS junction $^{2,3}$ and, in addition, when measured in magnetic field, they modulate in counter phase with respect to the $I_{C}$. Their presence offers a refined way to extract the junction parameters, such as dissipation and critical current density. ${ }^{18,25-28}$ In Fig. 5 we show a numerical simulation (hollow red dots) which well reproduces the experimental $I$ $-V$ curve. We used the well-known Sine-Gordon equation ${ }^{18}$ in presence of $0-\pi$ facets with a spatially dependent $j(x)$ current density ${ }^{31-36}$

$$
\varphi_{t t}-\varphi_{x x}+\alpha \varphi_{t}+\varepsilon(x) \sin \varphi=\gamma,
$$

with boundary conditions given in terms of normalized magnetic field $\eta$ 


$$
\varphi(0, t)=\varphi(l, t)=\eta .
$$

In Eq. (1), times are normalized to zero bias plasma frequency $\omega_{J}=\sqrt{2 \pi I_{0} / C \Phi_{0}}$ (with $I_{0}$ the critical current and $\Phi_{0}$ the flux quantum) and lengths to the Josephson penetration depth $\lambda_{J}=\sqrt{\Phi_{0} / 2 \pi \mu_{0} d J_{0}}$ (with $J_{0}=I_{0} / L=(1 / L) \int_{0}^{L}|j(x)| d x$ the mean absolute value of critical current density and $d=\lambda_{1}$ $\left.+\lambda_{2}+t\right)$. The coefficient $\varepsilon(x)=j(x) / J_{0}$ is related to the current distribution and is 1 for conventional facets and -1 for $\pi$ facets $^{31,32}$ and $\gamma=I_{b} / I_{0}$ is the normalized bias current. In Eq. (2), $l=L / \lambda_{J}$ is the normalized length of the junction, while the normalized magnetic field $\eta$ is given by $\left(2 \pi \mu_{0} \lambda_{L} \lambda_{J} / \Phi_{0}\right) B$, with $\alpha=1 / Q=1 / \sqrt{\beta_{C}}$ the normalized normal conductance and $\beta_{C}=2 \pi I_{0} R^{2} C / \Phi_{0}$ the StewartMcCumber parameter. ${ }^{18}$ The simulated curve reported in Fig. 5 was obtained for a two facets junction with $l=1.5$ and $\alpha$ $=0.28$. These fitting parameters condense the characteristic features we reproducibly found at the submicron scale: low dissipation $^{4,5}$ and relatively high $J_{C}$ values.

These $J_{C}$ values are higher than the average ones commonly reported for GB HTS junctions, ${ }^{2,3}$ but are consistent with the $d$-wave scenario in faceted junctions. For a reduced number of facets, as in this study, it is easier to create an unbalancement between 0 and $\pi$ facets and get closer to the real $J_{C}$ value, which would be measured only in the case of a single facet junction.

The enhancement of $J_{C}$, along with the high value of the London penetration depth of the (103) electrode, ${ }^{5}$ pushes the junctions toward the long regime $(l=1.5)$ even in the submicron scale. Therefore, resonance steps in the $I-V$ appear as evidence of semifluxon oscillation across the GB.

\section{CONCLUSIONS}

In conclusion we have shown how high quality submicron HTS junctions can be fabricated by using electron beam lithography and a carbon mask technique applied to the BP design. We have presented a systematic analysis of the transport properties of these devices and derived information on the barriers properties. A correlation between the $I_{C} R_{N}$ and the switching voltage has appeared consistently with a $d$-wave profile. This suggests a characteristic scaling energy which depends on the relative orientation of the $d$-wave electrodes. The low dissipation of the junctions and a much reduced number of facets also emerge as characteristic features.

Some corrections to the empirical laws regarding $J_{C}$ (for instance the relation with $I_{C} R_{N}, R_{N} A$, etc. ${ }^{2,3,37}$ ) might be expected once extrinsic microstructural effects are further reduced by the various nanotechnology recipes on the different types of HTS junctions. We believe also that the results presented here open the way to the ultimate target, i.e., reproducible, single facet junction a few hundreds nanometers wide. On this length scale, for our off-axis BP devices, we would have a single (103) oriented grain in the junction, and the advantages of an atomically flat basal plane GB could be fully exploited in more controllable devices.

\section{ACKNOWLEDGMENTS}

The authors would like to thank A. Barone, J. Kirtley and E. Goldobin for fruitful discussions. This work has been partially supported by the ESF Projects "MIDAS" and European Community FP6 transnational access Project "MC2ACCESS," Contract No. 026029.

${ }^{1}$ C. C. Tsuei and J. R. Kirtley, Rev. Mod. Phys. 72, 969 (2000).

${ }^{2}$ H. Hilgenkamp and J. Mannhart, Rev. Mod. Phys. 74, 485 (2002) (and references therein).

${ }^{3}$ F. Tafuri and J. R. Kirtley, Rep. Prog. Phys. 68, 2573 (2005).

${ }^{4}$ T. Bauch, F. Lombardi, F. Tafuri, A. Barone, G. Rotoli, P. Delsing, and T. Claeson, Phys. Rev. Lett. 94, 087003 (2005).

${ }^{5}$ T. Bauch, T. Lindstrom, F. Tafuri, G. Rotoli, P. Delsing, T. Cleason, and F. Lombardi, Science 311, 57 (2006).

${ }^{6}$ Y. V. Fominov, A. A. Golubov, and M. Yu. Kypryanov, JETP Lett. 77, 587 (2003); T. Yokoyama, S. Kawabata, T. Kato, and Y. Tanaka, Phys. Rev. B 76, 134501 (2007); M. H. S. Amin, A. Yu. Smirnov, A. M. Zagoskin, T. Lindstrom, S. A. Charlebois, T. Claeson, and A. Y. Tzalenchuk, ibid. 71, 064516 (2005)

${ }^{7}$ J. Clarke and F. K. Wilhelm, Nature (London) 453, 1031 (2008),

${ }^{8}$ F. Tafuri, F. Miletto Granozio, F. Carillo, A. Di Chiara, K. Verbist, and G. Van Tendeloo, Phys. Rev. B 59, 11523 (1999); A. Di Chiara, F. Lombardi, F. Miletto Granozio, U. Scotti di Uccio, F. Tafuri, and M. Valentino, IEEE Trans. Appl. Supercond. 7, 3327 (1997); F. Lombardi, T. Bauch, J. Johansson, K. Cedergren, T. Lindström, F. Tafuri, and E. Stepantsov, Physica C 435, 8 (2006).

${ }^{9}$ F. Lombardi, F. Tafuri, F. Ricci, F. Miletto Granozio, A. Barone, G. Testa, E. Sarnelli, J. R. Kirtley, and C. C. Tsuei, Phys. Rev. Lett. 89, 207001 (2002).

${ }^{10}$ K. Inomata, S. Sato, K. Nakajima, A. Tanaka, Y. Takano, H. B. Wang, M. Nagao, H. Hatano, and S. Kawabata, Phys. Rev. Lett. 95, 107005 (2005).

${ }^{11}$ A. Y. Tzalenchuk, T. Lindstrom, S. A. Charlebois, E. A. Stepantsov, Z. Ivanov, and A. M. Zagoskin, Phys. Rev. B 68, 100501 (2003).

${ }^{12}$ E. Il'ichev, M. Grajcar, R. Hlubina, R. P. J. IJsselsteijn, H. E. Hoenig, H.-G. Meyer, A. Golubov, M. H. S. Amin, A. M. Zagoskin, A. N. Omelyanchouk, and M. Yu. Kupriyanov, Phys. Rev. Lett. 86, 5369 (2001).

${ }^{13}$ F. Herbstritt, T. Kemen, L. Alff, A. Marx, and R. Gross, Appl. Phys. Lett. 78, 955 (2001).

${ }^{14}$ P. Larsson, B. Nilsson, and Z. G. Ivanov, J. Vac. Sci. Technol. B 18, 25 (2000).

${ }^{15}$ F. M. Granozio, U. S. di Uccio, F. Lombardi, F. Ricci, F. Bevilacqua, G. Ausanio, F. Carillo, and F. Tafuri, Phys. Rev. B 67, 184506 (2003).

${ }^{16}$ D. Stornaiuolo, K. Cedergren, D. Born, T. Bauch, A. Barone, F. Lombardi, and F. Tafuri, IEEE Trans. Appl. Supercond. 19, 174 (2009).

${ }^{17}$ P. V. Komissinski, B. Hogberg, A. Ya. Tzalenchuk, and Z. Ivanov, Appl. Phys. Lett. 80, 1022 (2002).

${ }^{18}$ A. Barone and G. Paterno, Physics and Applications of Josephson Effect (Wiley, New York, 1982).

${ }^{19}$ P. Dubos, H. Courtois, B. Pannetier, F. K. Wilhelm, A. D. Zaikin, and G. Schon, Phys. Rev. B 63, 064502 (2001); P. Dubos, H. Courtois, O. Buisson, and B. Pannetier, Phys. Rev. Lett. 87, 206801 (2001).

${ }^{20}$ L. Angers, F. Chiodi, G. Montambaux, M. Ferrier, S. Gueron, H. Bouchiat, and J. C. Cuevas, Phys. Rev. B 77, 165408 (2008).

${ }^{21}$ A. Tagliacozzo, D. Born, D. Stornaiuolo, E. Gambale, D. Dalena, F. Lombardi, A. Barone, B. L. Altshuler, and F. Tafuri, Phys. Rev. B 75, 012507 (2007).

${ }^{22}$ Z. G. Ivanov, E. A. Stepantsov, T. Claeson, F. Wenger, S. Y. Lin, N. Khare, and P. Chaudhari, Phys. Rev. B 57, 602 (1998).

${ }^{23}$ J. Mannhart, H. Hilgenkamp, B. Mayer, Ch. Gerber, J. R. Kirtley, K. A. Moler, and M. Sigrist, Phys. Rev. Lett. 77, 2782 (1996).

${ }^{24}$ P. A. Rosenthal, M. R. Beasley, K. Char, M. S. Colclough, and G. Zaharchuk, Appl. Phys. Lett. 59, 3482 (1991).

${ }^{25}$ J. Swihart, J. Appl. Phys. 32, 461 (1961).

${ }^{26}$ M. D. Fiske, Rev. Mod. Phys. 36, 221 (1964)

${ }^{27}$ R. E. Eck, D. J. Scalapino, and B. N. Taylor, Phys. Rev. Lett. 13, 15 (1964); A. Tagliacozzo, F. Tafuri, E. Gambale, B. Jouault, D. Born, P. Lucignano, D. Stornaiuolo, F. Lombardi, A. Barone, and B. L. Altshuler, Phys. Rev. B, 79, 024501 (2009).

${ }^{28}$ D. W. McLaughlin and A. C. Scott, Phys. Rev. A 18, 1652 (1978).

${ }^{29}$ C. Nappi, E. Sarnelli, M. Adamo, and M. A. Navacerrada, Phys. Rev. B 74, 144504 (2006); B. Chesca, R. R. Schulz, B. Goetz, C. W. Schneider, 
H. Hilgenkamp, and J. Mannhart, Phys. Rev. Lett. 88, 177003 (2002); E. Goldobin, D. Koelle, R. Kleiner, and A. Buzdin, Phys. Rev. B 76, 224523 (2007).

${ }^{30}$ J. T. Chen, T. F. Finnegan, and D. N. Langenberg, Physica (Utrecht) 55, 413 (1971); T. A. Fulton and R. C. Dynes, Solid State Commun. 12, 57 (1973); O. A. Levring, N. F. Pedersen, and M. R. Samuelsen, Appl. Phys. Lett. 40, 846 (1982); S. Pagano, M. P. Soerensen, P. L. Christiansen, and R. D. Parmentier, Phys. Rev. B 38, 4677 (1988).

${ }^{31}$ J. R. Kirtley, K. A. Moler, and D. J. Scalapino, Phys. Rev. B 56, 886 (1997).

${ }^{32}$ E. Goldobin, D. Koelle, and R. Kleiner, Phys. Rev. B 67, 224515 (2003).

${ }^{33}$ E. Goldobin, N. Stefanakis, D. Koelle, and R. Kleiner, Phys. Rev. B 70,
094520 (2004); T. Gaber, E. Goldobin, A. Sterck, R. Kleiner, D. Koelle, M. Siegel, and M. Neuhaus, ibid. 72, 054522 (2005).

${ }^{34}$ G. Rotoli, IEEE Trans. Appl. Supercond. 15, 852 (2005).

${ }^{35}$ E. Goldobin, K. Vogel, O. Crasser, R. Walser, W. P. Schleich, D. Koelle, and R. Kleiner, Phys. Rev. B 72, 054527 (2005); E. Goldobin, A. Sterck, T. Gaber, D. Koelle, and R. Kleiner, Phys. Rev. Lett. 92, 057005 (2004). ${ }^{36}$ N. Stefanakis, Phys. Rev. B 66, 214524 (2002); N. Lazarides, ibid. 69, 212501 (2004).

${ }^{37}$ R. Gross, P. Chaudhari, M. Kawasaki, and A. Gupta, Phys. Rev. B 42, 10735 (1990); S. E. Russek, D. K. Lathrop, B. H. Moeckly, R. A. Buhrman, D. H. Shin, and J. Silcox, Appl. Phys. Lett. 57, 1155 (1990). 